\title{
ACTIVIDAD INSECTICIDA DE EXTRACTOS ETANÓLICOS SOBRE Spodoptera frugiperda (Smith)
}

\section{INSECTICIDE ACTIVITY OF ETHANOLIC PLANT EXTRACTS ON Spodoptera frugiperda (Smith)}

\author{
iD Yvon Noguera ${ }^{1}$ \\ iD Pedro Morales Valles ${ }^{1}$ \\ ${ }^{1}$ Instituto Nacional de Investigaciones Agrícolas INIA, Venezuela.
}

Correspondencia:

Yvon Noguera

yvonjnoguera@gmail.com

\section{RESUMEN}

Se evaluó el efecto insecticida de extractos etanólicos de Neem (Azadirachta indica A. Juss), Tabaco (Nicotiana tabacum L), Anisote (Piper aduncum L.), Azahar de la india (Murraya paniculata) y Hierba mora (Solanum nigrum L.) sobre el gusano cogollero Spodotera frugiperda S. (Lepidoptera: Noctuidae), en condiciones de laboratorio. Se caracterizó el efecto de cuatro concentraciones de dichos extractos sobre la mortalidad de larvas y la emergencia de los adultos del insecto. Se evaluaron cinco tratamientos (extractos alcohólicos) más el testigo constituido por acetona. Se evaluaron 4 dosis con concentraciones de 250, 500, 750 y 1000 ppm. Se realizó una sola aplicación tópica de 5 microlitros de la dosis respectiva para cada larva del tercer instar, utilizando 20 larvas por repetición. Se utilizó un arreglo factorial 5×2, bajo un diseño en bloques al azar, con dos factores: los cinco extractos más el testigo y las cuatro concentraciones indicadas anteriormente para cada uno de los tratamientos. No hubo diferencias significativas entre tratamientos a nivel de mortalidad larval, caso contrario, en cuanto a emergencia de adultos, donde los tratamientos de Neem (45\%) y Anisote (43,75\%), se diferenciaron estadísticamente de la Hierba mora $(68,75 \%)$. Estos resultados indican el potencial uso del anisote y del Neem, para disminuir la emergencia de adultos en el campo.

Palabras clave: cogollero del maíz, insecticida orgánico vegetal, Neem

\section{ABSTRACT}

The insecticidal effect of ethanolic extracts of Neem (Azadirachta indica A. Juss), Tobacco (Nicotiana tabacum L.), Anisote (Piper aduncum L.), Indian orange blossom (Murraya paniculata) and Nightshade (Solanum nigrum L.) was evaluated on the fall armyworm Spodotera frugiperda S. (Lepidoptera: Noctuidae), under laboratory conditions. The effect of four concentrations of said extracts on the mortality of larvae and the emergence of the adults of the insect was characterized. Five treatments (alcoholic extracts) were evaluated plus the control constituted by acetone. 4 doses were evaluated with concentrations of $250,500,750$ and 1000 ppm. A single topical application of 5 microliters of the respective dose was made for each larva of the 
third instar, using 20 larvae per repetition. A 5x2 factorial arrangement was used, under a random block design, with two factors: the five extracts plus the control and the four concentrations indicated above for each of the treatments. There were no significant differences between treatments at the level of larval mortality, but there were significant differences in terms of adult emergence, where Neem (45 $\%)$ and Anisote (43.75 \%) treatments were statistically different from Nightshade (68.75\%). These results indicate the potential use of anisote and Neem to reduce the emergence of adults in the field.

Key words: armyworm, vegetal organic insecticide, Neem

\section{INTRODUCCIÓN}

El uso de plaguicidas sintéticos en la agricultura ha sido la forma más utilizada para la protección de los cultivos debido a la rapidez con que actúan, la eficiencia, fácil adquisición y transporte, además de su largo periodo de acción. Los productos sintéticos destinados a controlar plagas y enfermedades en los vegetales han tenido una incidencia muy marcada en el incremento de la producción agrícola (lannacone \& Lamas, 2003); sin embargo, esta eficacia en el control de plagas ha provocado serios daños a la agricultura: la resistencia de los organismos a los plaguicidas. (Anaya, 2003); la extinción de depredadores y parasitoides naturales, y de los polinizadores que forman parte del ecosistema (Freemark \& Boutin 1995) y el cambio de status de plagas consideradas ocasionales o de baja incidencia, a plagas importantes causantes de daños significativos en el cultivo por el aumento de su resistencia a los insecticidas (Hernandez et al., 2018).

Los Insecticidas vegetales son derivados - extraídos directamente de plantas y corresponden principalmente a mecanismos de defensa frente a posibles daños por insectos. Existe evidencia que los principios activos, se acumulan de distinta forma en una planta. El momento en que se encuentre la planta respecto a su ciclo de vida influyen en la cantidad de principios activos que contenga (Huerta, 2002).

Con el fin de, identificar nuevos productos fitotóxicos es importante realizar bioensayos con moléculas químicas de diversas especies de plantas y para ello es necesario el conocimiento de los compuestos presentes en ellas, lo cual se logra mediante el análisis de extracto de diferente polaridad obtenidos a partir de distintos tejidos y órganos (Reigosa et al., 2002).

El extracto vegetal es el resultado deconcentrar las sustancias activas y/o sustancias puras de una planta, donde se suele utilizar un vehículo como el etanol, para su obtención. Los extractos son utilizados en la industria farmacéutica y cosmetología, pero son difíciles de identificar, pueden contaminarse por el desarrollo de microorganismos, requiere de tiempo y de muchos pasos de filtración (Verbionat, 2008).

El gusano cogollero del maíz, Spodoptera frugiperda (Smith) (Lepidoptera: Noctuidae), es una de las plagas de mayor importancia en el cultivo del maíz. Lei et al. (2020), señalan que, este insecto presenta alta fecundidad, adaptabilidad y polifagia, atacando cultivos de importancia económica como el maíz, la soja y el sorgo, siendo una plaga grave en la mayor parte de América del Norte y del Sur. Recientemente se introdujo en África, Asia y Oceanía, donde ahora amenaza los cultivos en más de 110 países. En China, S. frugiperda se encontró por primera vez en la provincia de Yunnan en 2018, siendo una grave amenaza para su producción agrícola.

S. frugiperda tiene un ciclo de vida de huevo (de 3 a 5 días) 5 a 6 estados larvales diferenciables entre sí (de 14 a 21 días); pupa (de 9 a 13 días) y adulto (King \& Saunders, 1984). Este insecto puede causar daños en cualquier estadio del cultivo, dependiendo de la fecha de siembra y región; actuando como cortadora en los inicios del cultivo, como defoliadora dañando el cogollo en etapas vegetativas, incluso dañando el tallo como barrenadora en condiciones 
de sequía atacando la espiga en estadios reproductivos (MRI; IRAC-ARGENTINA, 2019).

Dos de las especies vegetales más utilizadas como insecticida vegetal es el Neem (Azadirachta indica A. Juss) cuyo componente, la azaridactina, es uno de los más estudiados y considerado como fitotóxico de amplio espectro, bajo efecto residual, sin toxicidad para los humanos y medio ambiente (García et al., 2017); y el tabaco (Nicotiana tabacum $L)$, contiene nicotina, que altera el sistema nervioso de los insectos conllevando a paro respiratorio y muerte (Manrique et al., 2017).

Otras especies vegetales con propiedades insecticidas son el Anisote (Piper aduncum L.) planta medicinal con usos antihemorrágicos (Aguirre et al., 2014). El compuesto más significativo de las plantas del género Piper es la piperamida piperina, que constituye el principio picante activo de la pimienta negra (Piper nigrum L.) y fitoquímicos como cromenos, fenilpropanoides, lignanos y neolignanos, alcaloides, y varios compuestos de origen biosintético mixto (Kato et al., 2007); el Azahar de la india (Murraya paniculata L.) Jack) presenta alcaloides, flavanoides, carotenoides, cumarinas y aceites esenciales (Rout et al., 2007) y la Hierba mora (Solanum nigrum L.), cuyos componentes activos saponinas, solaninas, y solanigrina, tienen un mayor efecto como repelente que como insecticida o antialimentario (Lizarazo et al., 2008).

Debido a que, el gusano cogollero, la principal plaga que afecta el cultivo del maíz, la aplicación de los extractos vegetales, elaborados con plantas o partes de ellas se presenta como una alternativa ecológicamente viable para reducir las poblaciones del insecto y disminuir las pérdidas en este cultivo. En atención a ello, esta investigación se condujo para evaluar in vitro la sobrevivencia larval y capacidad insecticida de cinco extractos etanólicos a base de Neem (Azadirachta indica), Tabaco (Nicotiana tabacum), Anisote (Piper aduncum), Azar de la india (Murraya paniculata) y Hierba mora (Solanum nigrum) sobre el gusano cogollero del maíz (S. frugiperda)

\section{MATERIALES Y MÉTODOS}

El ensayo se realizó en las instalaciones de los laboratorios de Bacteriología y Entomología del edifico 2 del Centro Nacional de Investigaciones Agropecuarias (CENIAP) del Instituto Nacional de Investigaciones Agrícola (INIA), Las coordenadas del lugar son: Latitud de 10 $17^{\prime}$ logitud de 67은 y altitud de 48 m s.n.m. Ubicado en el Área Universitaria en Maracay, estado Aragua, Venezuela.

Los materiales evaluados fueron: Neem (Azadirachta indica A. Juss), Tabaco (Nicotiana tabacum L), Anisote (Piper aduncum L.), azahar de la india (Murraya paniculata (L.) Jack.); hierba mora (Solanum nigrum L.).

El Neem y el Azahar de la India fueron colectados en el Campo Experimental del INIACENIAP, Maracay, estado Aragua; el tabaco en Montalbán, estado Carabobo; la hierba mora en las inmediaciones de la Universidad Central de Venezuela, Facultad de Agronomía, Maracay; y el Anisote en Ocumare de la costa, estado Aragua. Se usaron las hojas hasta tener una mezcla completa. Las colectas se realizaron durante el período de agosto-septiembre del año 2009.

El material vegetal colectado se caracterizó por tener hojas bien desarrolladas, libres de manchas y perforaciones por insectos. Fueron secadas en umbráculo, sobre mesones de concreto a temperatura ambiente $(44 \pm 2 \stackrel{\circ}{\circ} \mathrm{C}, 36$ $\% \pm \mathrm{HR}$ ) durante 7 a 10 días.

Se pesaron 500 gr de cada material vegetal, triturado y pulverizado con ayuda de una licuadora marca Oster, con el fin de, obtener trozos del material de tamaño homogéneo. Posteriormente se procedió a la inmersión del material vegetal en etanol al $98 \%$, hasta completar la capacidad disponible del envase, se dejó reposar la mezcla durante siete días en oscuridad con el objeto de que el etanol extrajera los metabolitos solubles presente en cada especie vegetal. En la Tabla 1, se indican las especies utilizadas para extraer los extractos etanólicos. 
Tabla 1

Especies de plantas, peso del material seco y volumen de alcohol utilizados para la obtención de los extractos en el ensayo

\begin{tabular}{|c|c|c|c|}
\hline $\begin{array}{l}\text { Nombre común de } \\
\text { planta }\end{array}$ & Nombre científico & $\begin{array}{l}\text { Peso material } \\
\text { seco }(\mathrm{gr})\end{array}$ & $\begin{array}{c}\text { Volumen de alcoho } \\
\text { usado (L) }\end{array}$ \\
\hline Neem & $\begin{array}{l}\text { (Azadirachta indica A. } \\
\text { Juss), }\end{array}$ & 500 & 3500 \\
\hline Azahar de la india & $\begin{array}{l}\text { (Murraya paniculata (L.) } \\
\text { Jack }\end{array}$ & 500 & 3500 \\
\hline Tabaco & (Nicotiana tabacum L & 500 & 3480 \\
\hline Anisote & (Piper aduncum L.), & 500 & 3500 \\
\hline Hierba mora & (Solanum nigrum L.). & 500 & 3500 \\
\hline
\end{tabular}

Para la obtención de los extractos etanólicos de las diferentes especies vegetales se siguió el protocolo propuesto por Marcano \& Hasegawa (2002). Siguiendo este método, se realizaron los siguientes pasos: 1.- Se dejó macerar por un periodo de 48 a $72 \mathrm{~h}$ en un lugar fresco y oscuro; 2.- Se filtró a través de cuatro capas de gasas, para la extracción del soluto; 3.- El Filtrado fue llevado a rotoevaporador (marca Yamato modelo BM 200) a temperatura de 50 ${ }^{\circ} \mathrm{C}$ y 100 rpm durante 40-45 min. dependiendo del material vegetal y 4.- El material obtenido se conservó en frascos estériles de color ámbar, previamente etiquetados, para su conservación bajo refrigeración a una temperatura de $4^{\circ} \mathrm{C}$, hasta el momento que se realizó el ensayo.

Para la obtención de crías del insecto, se colectaron larvas de cogollero en plantaciones de maíz con suficiente material para la emergencia de adultos, los cuales se colocaron aparte en envases de vidrio de boca ancha con una capacidad de $3,750 \mathrm{~L}$, donde previamente se colocó una toalla de papel absorbente como sitio de ovoposición y reposo.

Estos frascos fueron colocados en un estante oscuro y se le colocó una esponja de algodón en agua de azúcar al 1 \%, la cual permitió que los adultos se alimentaran, luego se taparon los frascos con servilletas, sujetas con una banda elástica de goma.

Los huevos depositados en el papel absorbente se retiraron del frasco cada dos días para así obtener las larvas. El papel contenido con las posturas se cortó en secciones tratando de quitarle el máximo del papel, colocándose en frascos con su respectiva dieta artificial y se tapó con una toalla absorbente sujeta mediante una goma elástica. Al cabo de tres días aproximadamente, se observó la eclosión de las larvas. Separándose las larvas del segundo instar, para evitar el canibalismo entre las misma. Posteriormente, se ubicó cada espécimen en envases plásticos, marca selva, transparente con una capacidad de $25 \mathrm{ml}$ con sus respectivas tapas plástica, colocando su respectiva dieta artificial, el trozo de dieta con $1 \mathrm{~m}^{3}$ para evitar incremento de la humedad dentro del recipiente.

La cría de S. frugiperda se llevó a cabo, según lo propuesto por García (1982). La dieta estuvo compuesta por los siguientes ingredientes: $400 \mathrm{~g}$ de caraotas blancas, 60 $\mathrm{g}$ de levadura, $6 \mathrm{~g}$ de ácido ascórbico, $4 \mathrm{~g}$ de metil-p-hidroxibenzoato (diluidos en $10 \mathrm{ml}$ de alcohol puro), $2 \mathrm{~g}$ de ácido sórbico, $4 \mathrm{ml}$ de formol, $500 \mathrm{ml}$ de agua, $500 \mathrm{ml}$ de agua para el agar, $30 \mathrm{~g}$ de agar. El procedimiento fue el siguiente:

Se cocinaron las caraotas hasta que se ablandaran, luego se colocaron los $500 \mathrm{ml}$ de agua en el homogeneizador y se añadieron todos los ingredientes, menos el agar y las caraotas, posteriormente, se homogeneizó por 30 segundos. Luego, se añadieron las caraotas y se homogeneizó primero por un minuto y después, por 30 segundos. En otro recipiente se calentaron los $500 \mathrm{ml}$ de agua hasta una temperatura de $80{ }^{\circ} \mathrm{C}$, en donde se añadió lentamente el agar removiendo constantemente; una vez disuelto el agar, se 
añadió a la mezcla anterior y se homogeneizó por un minuto. La mezcla fue vertida en una bandeja para su enfriamiento a temperatura ambiente. Una vez atemperada la dieta, ya en estado sólido, se cortó en cubos de 0,8 mm y se colocaron con las larvas en los envases.

El experimento se realizó, por medio de un diseño factorial $5 \times 2$ en bloques al azar con 5 tratamientos (extractos de plantas) y 5 niveles (dosis) correspondientes a b0: 1000 ppm, b1: 750 ppm, b2: 500 ppm, b3: 250 ppm y b4: 0 ppm (testigo con acetona). Para los bloques se consideró, el efecto de las mediciones en el tiempo (3 días), ya que las observaciones se obtienen de la medición del número de larvas muertas a las 24 h (un día), 48 h (2 días) y 72 h (3 días), y se mantuvieron en observación hasta llegar a la fase adulta, en condiciones de laboratorio a temperatura de $28 \pm 2{ }^{\circ} \mathrm{C}, \mathrm{HR}$ $75 \pm 5 \%$ y Fotoperiodo 12:12.

En el pronoto de cada larva del cuarto al quinto instar se aplicó una dosis de 5 microlitros de manera tópica, con la respectiva concentración. La unidad experimental fue de 20 larvas, para un total de 500 larvas usadas en el experimento.

Las observaciones se realizaron a las 24 , 48 y 72 h, posterior a la aplicación de los tratamientos, para el registro de larvas muertas. Posteriormente, se llevó el seguimiento de las larvas que sobrevivieron y continuaran su desarrollo hasta la emergencia de los adultos para su contabilización. Los factores y niveles de extractos etanólicos considerados se muestran en la Tabla 2.

Tabla 2

Factores y niveles de extractos etanólicos

\begin{tabular}{llll}
\hline Factor & Niveles & Factor & Niveles \\
\hline A: Extracto & $\mathrm{a}_{0}$ : Extracto de Azahar de la India & B: Dosis & $\mathrm{b}_{0}: 1000 \mathrm{ppm}$ \\
& $\mathrm{a}_{1}:$ Extracto de Neem & & $\mathrm{b}_{1}: 750 \mathrm{ppm}$ \\
& $\mathrm{a}_{2}:$ Extracto de Hierba Mora & $\mathrm{b}_{2}: 500 \mathrm{ppm}$ \\
& $\mathrm{a}_{3}:$ Extracto de Tabaco & $\mathrm{b}_{3}: 250 \mathrm{ppm}$ \\
& $\mathrm{a}_{4}:$ Extracto de Anisote & $\mathrm{b}_{4}: 0 \mathrm{ppm}$ \\
\hline
\end{tabular}

Se empleó un Análisis de Varianza (Anova), para un arreglo factorial con bloques al azar con nivel de significancia de $5 \%$. No se realizó la corrección y/o evaluación de la mortalidad en base a los diferentes extractos promulgada por Abbot, ya que su aplicación se realiza cuando la mortalidad en el testigo es de un 5 al 20 \% (Saume, 1992) y en el ensayo se obtuvo un $0 \%$ de mortalidad en el testigo. Se efectuaron pruebas de normalidad y homocedasticidad de varianzas por medio del programa estadístico SAS (Statistical Analysis System), para caracterizar los supuestos y como resultado del mismo se procesaron los datos en el programa Statistix 8.0 y se realizó la prueba de Kruskal-Wallis para datos no paramétricos y las pruebas de medias para las variables concentración y tiempo entre las especies vegetales.

\section{RESULTADOS Y DISCUSIÓN}

En la Tabla 3, se presentan los promedios de larvas vivas a las 24, 48 y 72 h, después de aplicados los tratamientos. Aunque hubo alta sobrevivencia larval, se pudo observar menor promedio de larvas vivas a las $72 \mathrm{~h}$. Con respecto a las 48 y $72 \mathrm{~h}$, hubo diferencias significativas en cuanto al promedio de larvas. 
Tabla 3

Promedios de larvas vivas totales a las 24,48 y $72 \mathrm{~h}$ después de aplicados los tratamientos

\begin{tabular}{cc}
\hline Tiempo & Larvas \\
\hline 24 & $99,28^{\mathrm{a}}$ \\
48 & $98,81^{\mathrm{a}}$ \\
72 & $96,67^{\mathrm{b}}$ \\
\hline Letras distintas indican diferencias sionificativas $(\mathrm{p}<0,05)$
\end{tabular}

En la Tabla 4, se observan los porcentajes de larvas vivas a las $72 \mathrm{~h}$ de aplicados los tratamientos en las diferentes dosis utilizadas. Los valores de sobrevivencia larval fueron altos en todos los tratamientos utilizados.
Esto indica el bajo efecto agudo para causar la mortalidad de larvas por dichos extractos. El extracto de tabaco presentó la menor sobrevivencia larval $(19,17)$ diferente del resto de los tratamientos.

\section{Tabla 4 \\ Porcentaje de larvas vivas por tratamiento a las $72 \mathrm{~h}$ de aplicación}

\begin{tabular}{cc} 
Tratamientos & Larvas vivas \% \\
\hline Testigo & $100^{\mathrm{a}}$ \\
Neem & $100^{\mathrm{a}}$ \\
Anisote & $99,58^{\mathrm{a}}$ \\
Azahar & $98,33^{\mathrm{ab}}$ \\
Hierba Mora & $97,10^{\mathrm{ab}}$ \\
Tabaco & $95.83^{\mathrm{b}}$ \\
\hline Letras distintas indican diferencias significativas $(\mathrm{p}<0,05)$
\end{tabular}

Barón \& Angulo (2003), refiere que, al mezclar hojas secas de tabaco con agua y vinagre, para luego ser asperjado al cultivo de maíz en $h$ de la mañana o en la tarde se observó como resultado del 90 al $100 \%$ de mortalidad en larvas de Spodoptera.

Romero (2015), obtuvo valores de mortalidad elevados (55-92.5\%) utilizando extractos etanólicos de Lantana cámara para el control de moscas blancas al conducir investigaciones en condiciones de laboratorio.

En nuestro caso, habría que considerar una probable disminución de la susceptibilidad de esta selección de las poblaciones de $S$. frugiperda a los insecticidas, en virtud que las larvas fueron colectadas de una zona de alto uso de agroquímicos y la edad de las larvas (cuarto al quinto instar) lo que pudo hacerlas menos susceptibles a los productos utilizados. García et al. (2012), señala que utilizaron $1 \mu \mathrm{L}$ por dosis de insecticida, pero en larvas del tercer instar.

En la Tabla 5, se aprecia un mínimo efecto en la baja toxicidad aguda de los mismos para causar la mortalidad de las larvas. El extracto de Hierba mora presentó diferencias significativas con relación al resto de los tratamientos a las $72 \mathrm{~h}$ después de aplicación 
Tabla 5

Mortalidad de larvas vivas por tratamiento a las 24,48 y $72 \mathrm{~h}$

\begin{tabular}{cccc}
\hline & \multicolumn{3}{c}{ Tiempos } \\
Larvas & 24 & 48 & 72 \\
\hline Testigo & $100^{\mathrm{a}}$ & $100^{\mathrm{a}}$ & $100^{\mathrm{a}}$ \\
Neem & $100^{\mathrm{a}}$ & $100^{\mathrm{a}}$ & $100^{\mathrm{a}}$ \\
Anisote & $100^{\mathrm{a}}$ & $100^{\mathrm{a}}$ & $98,75^{\mathrm{ab}}$ \\
Azahar & $100^{\mathrm{a}}$ & $98,75^{\mathrm{ab}}$ & $96,25^{\mathrm{ab}}$ \\
Hierba mora & $100^{\mathrm{a}}$ & $98,75^{\mathrm{ab}}$ & $92,50^{\mathrm{ab}}$ \\
Tabaco & $96,25^{\mathrm{ab}}$ & $96,00^{\mathrm{ab}}$ & $95,00^{\mathrm{ab}}$ \\
\hline
\end{tabular}

Letras distintas indican diferencias significati vas $(\mathrm{p}<0,05)$

En la Figura 1, se observa el número de adultos emergidos por tratamiento aplicado en cada una de las dosis respectivas. Se aprecia la tendencia general que a mayor dosis del extracto menor número de adultos emergidos, a excepción de hierba mora, el cual fue el tratamiento con el menor efecto toxico en cuanto a emergencia de los individuos evaluados.

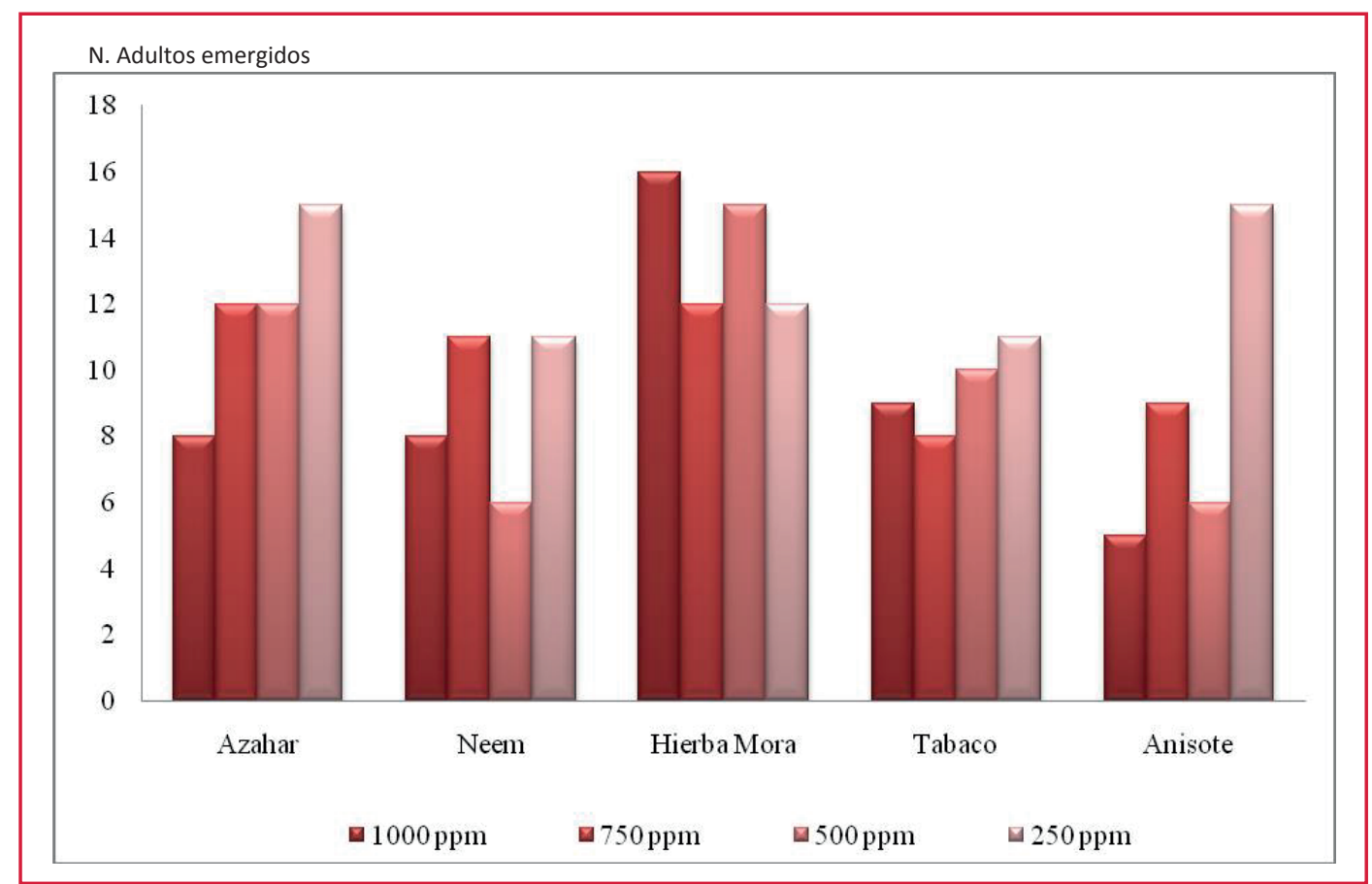

Figura 1. Número de adultos emergidos de S. frugiperda (Smith) en función de la acción de los extractos etanólicos de cinco especies botánicas en laboratorio.

Molina (2001), señala que el extracto de Neem cuyo ingrediente activo es la azadirachtina, presenta un efecto insecticida sobre estados inmaduros (larvas, ninfas y pupas) de algunos insectos plaga (lepidópteros, afidios, mosca blanca y ácaros). 


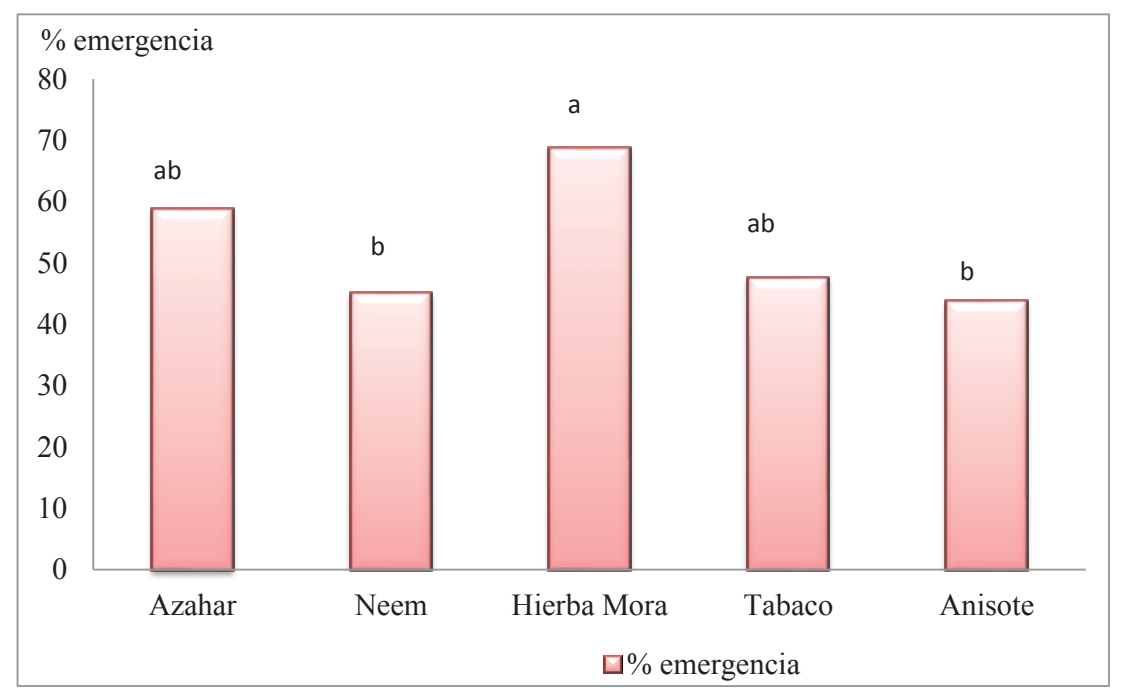

Figura 2. Porcentaje de emergencia de adultos de Spodoptera frugiperda (Smith) en función de la acción de los extractos etanólicos de cinco especies botánicas. T: $28 \pm$ $2^{\circ} \mathrm{C}, \mathrm{HR}: 75 \pm 5 \%$, fotoperiodo 12:12.

En la Figura 2, se presentan los porcentajes de emergencia de adultos de S. frugiperda para cada uno de los tratamientos aplicados. Se aprecia como los tratamientos que presentaron una menor emergencia de adultos fueron los tratamientos a base de Neem y Anisote, cuyos valores se diferenciaron estadísticamente de la Hierba mora, que presentó el mayor porcentaje de emergencia de adultos.

Estos resultados de mortalidad contrastan con lo observado en las larvas, donde los valores de sobrevivencia fueron elevados al observar los tratamientos con base de extractos de Neem y Anisote.

Estos valores porcentuales de emergencia de adultos nos permiten inferir que los mencionados extractos, aplicados para controlar larvas de S. frugiperda podrían presentar un efecto negativo en la emergencia de poblaciones de este insecto a largo plazo inhibiendo su metamorfosis e impidiendo la emergencia de los adultos.

Los extractos de Neem y cariaquito han resultado exitosos en el caso de A.gossypii, como señala Montero et al. (2017), donde la mortalidad acumulada en función del tiempo tiende a aumentar a medida que se utilizan mayores concentraciones de los extractos evaluados y en el caso del Neem y Cariaquito, se obtuvieron valores de mortalidad del 98 y $100 \%$. Lo cual confirman la posible utilidad del extracto Neem para controlar las poblaciones de S. frugiperda en siembra de maíz.

\section{CONCLUSIONES}

- No se detectó efecto insecticida de los extractos evaluados en larvas del gusano cogollero, en función de las concentraciones y fase larval utilizadas en el ensayo.

- Los extractos etanólicos evaluados, mostraron efecto insecticida al disminuir la emergencia de adultos del insecto. Se pueden considerar como promisorios en este aspecto, los extractos etanólicos de hojas de Neem y Anisote, para disminuir la emergencia de adultos.

- Se apreció la tendencia general que, a mayor dosis del extracto aplicado menor número de adultos emergidos, a excepción de hierba mora, el cual fue el tratamiento con el menor efecto toxico en las concentraciones utilizadas.

\section{REFERENCIAS BIBLIOGRÁFICAS}

Aguirre, Z., Yaguana C. \& Merino B. (2014). Plantas medicinales de la zona andina de la provincia de Loja. Primera Edición. Loja, Ecuador. 193 p.

Anaya L. (2003). Ecología química. 
México DF, Plaza y Valdéz, 349 p.

Barón, F. \& Angulo, J. (2003). El gusano cogollero del maíz, (Spodoptera frugiperda Smith). AGROSAVIA, Corpoica. Colombia. 25 p. Disponible en: http://hdl. handle.net/20.500.12324/2220

Freemark K., \& Boutin C. (1995). Impacts of agricultural herbicide use on terrestrial wildlife in temperate landscapes: A review with special reference to North America. Agriculture, Ecosystems \& Environment, 52(2-3), 67-91. DOI: https:// doi.org/10.1016/0167-8809(94)00534-L

García, J. (1982). Estudio sobre la biología, comportamiento y ecología de Spodoptera frugiperda (Smith) (Lepidoptera: Notuidae) [Tesis de doctorado]. Universidad Central de Venezuela, Maracay, Venezuela. 222 pp.

García, I., Rodríguez, E., Ortega, L., Solís, J. (2012). Susceptibilidad de Spodoptera frugiperda (J. E. Smith) (Lepidoptera: Noctuidae) a insecticidas asociada a césped en Quintana Roo, México. Agrociencia, 46(3), 279-287. Disponible en: $\quad$ http://www.scielo.org.mx/scielo. php? script=sci_arttext $\&$ pid $=S 1405$ $31952012000300007 \&$ lng=es\&tlng=es.

García, Y., Castro, M., López, M., Cardenas, E., \& Molina, R. (2017). Efecto del extracto de hoja de Neem (Azadirachta indica) para control de ectoparásitos en perros. Revista Científica, XXVII(3),154-161. Disponible en: https://www.redalyc.org/articulo. oa? id=95952010004

Hernández, A., Osorio, E., López, J., Ríos C., Varela, S., \& Rodríguez, R. (2018). Insectos benéficos asociados al control del gusano cogollero (Spodoptera frugiperda) en el cultivo de maíz (Zea mays L.). Agro Productividad. 1(11), 9-14. Disponible en: https://revistaagroproductividad.org/index.php/ agroproductividad/article/view/142
Huerta, A. (2002). Propiedades Insecticidas del Árbol del Paraíso (Melia azedarach L.). Disponible en: www. forestal.uchile.cl/ambiente_forestal/ ambiente_forestal_3/cap5.pdf.: 25-31

lannacone, J., \& Lamas, G. (2003). Efecto insecticida de cuatro extractos botánicos y del cartap sobre la polilla de la papa Phthorimaea operculella (Zeller) (Lepidoptera: Gelechiidae), en el Perú. Entomotrópica, 18(2), 95-105. Disponible en: https://dialnet.unirioja.es/servlet/ articulo? codigo $=717264$

Kato, M. \& Furlan, M. (2007). Chemistry and evolution of the Piperaceae. The Journal Pure and Applied Chemistry. 79(4). 529-538. DOl: https://doi. org/10.1351/pac200779040529

King, A., \& Saunders, L. (1984). Las plagas invertebradas de cultivos alimenticios anuales en América Central. Administración de Desarrollo Extranjero Londres; Overseas Development Administration. $182 \mathrm{p}$.

Lei, Ch., Yang J., Wang J., Hu J., \& Sun X. (2020). Molecular and Biological Characterization of Spodoptera frugiperda Multiple Nucleopolyhedrovirus Field Isolate and Genotypes from China. Insects, 11(11), 777. DOl: https://doi. org/10.3390/insects 11110777

Lizarazo, K., Mendoza, C. \& Carrero, R. (2008). Efecto de extractos vegetales de Polygonum hydropiperoides, Solanum nigrum y Calliandra pittieri sobre el gusano cogollero (Spodoptera frugiperda). Agronomía Colombiana, 26(3), 427-434. Disponible en: https:// revistas.unal.edu.co/index.php/agrocol/ article/view/11474

Manrique J, Eslava I, \& Chaparro J. (2017). Uso integral de colillas de cigarrillo con fines ambientales y comerciales. Proyecto piloto en la facultad del medio ambiente 
de la universidad distrital Francisco José de Caldas. Boletín Semillas Ambientales, 11(1), 72-79. Disponible en: https:// revistas.udistrital.edu.co/index.php/bsa/ article/view/12234

Marcano, D. \& Hasegawa, M. (2002). Fotoquímica Orgánica. Segunda edición. Editorial Torino. Caracas, Venezuela. 602 p.

Molina, N. (2001). Uso de extractos botánicos en control de plagas y enfermedades. Manejo Integrado de Plagas. 59,76-77. Disponible en: https:// biblat.unam.mx/es/buscar/uso-deextractos-botanicos-en-control-deplagas-y-enfermedades

Montero, O., Morales, P., Pino, O., Cermeli, M., González, E., \& Rosales, L. (2017). Actividad insecticida de seis extractos vegetales sobre Aphis gossypii Glover (Hemiptera: Aphididae). Revista de Protección Vegetal, 32(3): 1-7. Disponible en: http://scielo.sld.cu/scielo. php? script $=$ sci_arttext $\&$ pid $=S 1010$ $27522017000300006 \&$ lng=es\&tlng=es.

Programa Manejo de Resistencia de Insectos (MRI) e Comité de Acción de Resistencia a Insecticidas (IRACArgentina) (2019). Cogollero (Spodoptera frugiperda) en el cultivo de maíz Bases para su manejo y control en sistemas de producción. Disponible en: https:// aapresid.org.ar/wp-content/uploads/ sites/3/2019/12/Cogollero-1.pdf

Reigosa, M., \& Pedrol, N. (2002). Allelopathy frommolecules to ecosystems. Sciencie Publisher, inc. USA.INC 3/6.

Romero, R. Morales P, Pino O, Cermeli M, \& González E. (2015). Actividad insecticida de seis extractos etanólicos de plantas sobre mosca blanca. Revista de Protección Vegetal, 30(1),11-16. Disponible en: http://scielo.sld.cu/scielo. php? script $=$ sci_arttext \&pid $=$ S1010 $27522015000400005 \&$ lng $=$ es \& tlng $=e$
Rout, P., Rao, Y., \& Sree A. (2007). Composition of essential oil, concrete, absolute, wax and headspace volatiles of Murrarya paniculata (Linn.) Jack flowers. Flavour and Fragrance Journal, 22(5), 352-357. DOI: https://doi.org/10.1002/ ffj.1804

Saume, F. (1992). Introducción a la Química y Toxicólogoía de insecticidas (Industria Gráfica Integral). Aragua, Venezuela, 184-206.

Verbionat (2008). Extractos vegetales. Disponible en: http://www.verbionat. com/labs/index.php?option=com content\&task=view\&id=95\&ltemid $=112$ 